\title{
Telemedicine Services in the University of the Philippines Health Service during the COVID-19 Pandemic: A Two-week Process Documentation and Analysis
}

Geannagail O. Anuran, MD, Katrina Lenora Villarante, MD, Marishiel D. Mejia-Samonte, MD, Theresa A. Villa, MD, AM. Karoline V. Gabuyo, MD, Kashmir Mae B. Engada, MD, Jonathan D. Babsa-ay, MD and Shiela Marie S. Laviña, MD

Department of Family and Community Medicine, Philippine General Hospital, University of the Philippines Manila

\begin{abstract}
Background. Telemedicine provides access to health care services during pandemics. It can be utilized to screen asymptomatic persons, follow up close contacts of confirmed cases, monitor individuals with symptoms, conduct specialty consultations, and offer health services to patients during pandemics.
\end{abstract}

Objective. To describe the telemedicine processes, good practices, and areas for improvement in the University of the Philippines Health Service (UPHS) during the COVID-19 pandemic.

Methods. This was a cross-sectional study to document telemedicine processes in UPHS. All teleconsultations of employees and students of Philippine General Hospital (PGH) and UP Manila (UPM) during the two-week study period in October 2020 were included. Quantitative data was collected from different modes of patient entry into the UPHS telemedicine services: email, Online Consultation Request and Appointment (OCRA) System, and phone hotlines. Qualitative information was gathered as narrative descriptions of observations in the clinic's service delivery areas. A focus group discussion was also conducted to illustrate the different steps of the pathway used for telemedicine.

Results. The telemedicine services of UPHS consisted of virtual triage, COVID-19/non-COVID-19 consultation, and telemonitoring. The UPHS virtual triage received patient concerns through OCRA or the hotline numbers. On the other hand, the COVID-19 teleconsultation service provided care to employees and students who contacted the clinic regarding symptoms or exposure via email. The non-COVID-19 service had teleconsultation for patients with other medical concerns. Coordination among staff and presence of a consultant were identified as good practices, while the areas for improvement include the lack of written protocols in issuing fit-to-work clearance for difficult cases and the optional use of OCRA for UPHS consult.

Conclusion. Telemedicine services at the UPHS included tele-triaging, teleconsultations, and telemonitoring with use of phone calls, short messaging service (SMS), emails, and OCRA. Timely coordination, on-site duty consultants, and use of technology were identified as good practices. Lack of protocols and inconsistent OCRA use are areas for improvement.

Key Words: employees' clinic, pandemic, telemedicine

Poster presented in the $16^{\text {th }}$ Science and Technology Week Digital Poster Presentation on November 23 to December 4, 2020, at the University of the Philippines Manila, Philippines.

Corresponding author: Geannagail O. Anuran, MD

Department of Family and Community Medicine

Philippine General Hospital

University of the Philippines Manila

Taft Avenue, Ermita, Manila 1000, Philippines

Email: goanuran@up.edu.ph

\section{INTRODUCTION}

Telemedicine provides access to health care services during pandemics. It can be a safe environment for patienthealth provider relationships when physical consultation is not possible. ${ }^{1}$ Likewise, different telemedicine services can be utilized to screen asymptomatic persons, follow-up case contacts, monitor symptomatic cases, perform specialty 
consultations, and allow patients to access various health services even under quarantine. ${ }^{2}$

The University of the Philippines Health Service (UPHS) is the outpatient clinic for Philippine General Hospital (PGH) staff, UP Manila (UPM) employees and students. The clinic's daily operations, processes of consultations and other services were drastically changed by the COVID-19 pandemic. Telemedicine was incorporated in the UPHS service delivery processes to augment clinic capacity for consultations, while maintaining physical distancing and limiting exposure to infections.

Voice calls, text messaging and e-mail messages have been part of the teleconsultation process together with various digital applications used for telemonitoring of COVID-19 contacts and suspects. These communication tools automated and expanded several functions of UPHS. However, there was also a frequent need to modify processes within the clinic due to COVID-19-related circumstances and policy changes. The constant realignment of telemedicine services in the delivery of healthcare at the UPHS led to a process documentation and analysis that identified good practices and areas needing improvement. Hence, the general objective of the study was to describe the telemedicine health delivery processes, the good practices, and areas for improvement at the UP Health Service during the COVID-19 pandemic.

\section{METHODS}

\section{Study Design and Population}

This was a cross-sectional study involving process documentation and analysis of telemedicine health delivery at the UPHS. All teleconsultations by PGH health care workers (HCW), UPM employees and students for COVID-19, other medical concerns, periodic health examination, and monitoring in the last two weeks of October 2020 during the COVID-19 pandemic were included. On the other hand, we excluded all inquiries which did not lead to a triage assessment or a teleconsultation, and concerns from non-UPM/PGH employees/students, or from UP system constituents for nonUPHS patients such as family members or friends.

\section{Sample Size and Sampling}

There was no sample size computation and sampling. All teleconsultation of PGH HCWs, UPM employees and students during the study period were included.

\section{Data Collection Procedure}

Data was collected from the different telemedicine services at the start and end of each clinic day. Data collection forms for both the qualitative and quantitative parts of the study were pilot-tested. Narrative descriptions of observations and problems in the clinic's service delivery processes were also collected daily. These included process flows, issues encountered, communication strategies, staffing, variations from the standard process flows, and good practices observed.
Good telemedicine practices were any customary, habitual, or expected procedure or way of doing something that has been shown to be effective in UPHS in terms of being able to 1) decrease the learning curve of physicians who are new to UPHS; 2) reduce rework; 3) improve productivity and efficiency; 4) improve services; 5) and minimize knowledge loss. These criteria were adapted from those written by Olivier Serrat for the Asian Development Bank. ${ }^{3}$

A focus group discussion (FGD) about the actual steps of the UPHS processes was conducted among consultants, residents and nursing staff in order to illustrate the different process pathways. Three physicians were FGD facilitators, while one research assistant functioned as a scribe. Eleven UPHS staff were part of the focus groups. Two nurses illustrated the virtual triaging, two consultants discussed the non-COVID-19 teleconsultation process, two senior residents were assigned to telemonitoring, and seven junior residents described the COVID-19 teleconsultation and periodic health examination processes.

Data sources included the web-based Computerized Registry of Admissions and Discharges (RADISH), online documentation database for case investigation form (CIF) and Real time-Polymerase chain reaction (RT-PCR) results (Open ERP), official UPHS email, Online Consultation Request and Appointment (OCRA) System, UPHS teleconsult census list, and the Google Sheet spreadsheet Telemonitoring database.

\section{Study Outcomes and Data Analysis Plan}

Study outcomes included patient load for emails, OCRA, hotline numbers and telemonitoring service; diagrams of UPHS telemedicine services: triage, COVID-19/NonCOVID-19 teleconsult, and telemonitoring; and narrative descriptions on UPHS telemedicine processes, areas for improvement, good practices facilitating process flow and patient care.

Quantitative data was encoded and analyzed using Microsoft Excel Version 16.16.21. Descriptive statistics such as mean, standard deviations and median was used to summarize numerical data, while qualitative data underwent content analysis through segment coding in MAXQDA analytic pro software.

\section{Ethical Considerations}

The study proposal was approved by the Ethics Review Board of the University of the Philippines-Philippine General Hospital.

The study procedures (data collection, data entry and analysis) were in accordance with the Implementing Rules and Regulations of the Data Privacy Act of 2012. Data from the individual employees and students who were seen by the telemedicine service were aggregated as counts and were not identifiable in the data collection forms. There was no direct participation from the patients during the conduct of the study. 


\section{Waiver of Informed Consent among UPHS patients who used telemedicine}

The study investigators requested a waiver of informed consent as the study involved an analysis of the different repositories of data and did not adversely affect the rights and welfare of health workers, university employees and students.

\section{RESULTS}

A total of 3,991 counts of telemedicine services were provided by the UPHS during the two-week study period. The average daily service hours ranged from 5.5 hours for the COVID-19 teleconsultation service to 7 hours and 43 minutes for the virtual triage.

The telemedicine services of UPHS consisted of virtual triage, COVID-19 and non-COVID-19 related consultation and telemonitoring (Figure 1). The virtual triage received patient concerns through OCRA or the official UPHS hotline mobile and telephone numbers. On the other hand, the COVID-19 teleconsultation service provided care to employees/students who initially contacted the clinic regarding symptoms or exposure via email and were subsequently enrolled into the telemonitoring service. The nonCOVID-19 service had teleconsultation for patients who requested an appointment through OCRA or the hotline.

\section{UPHS Virtual Triage}

The virtual triage received a total of 698 texts, 261 appointment requests and 129 voice calls (Table 1). There was a daily average of 10 calls (SD 5.2) and 50 text messages (SD 23.3) with a maximum of 93 messages. Nurses triaged employees or students into the appropriate sub-clinic based on concerns (Figure 2).

Employees and students with COVID-19 symptoms or community exposure to a confirmed case were advised to send a CIF to the UPHS email for a teleconsultation and swab scheduling. COVID-19-related calls averaged six (SD 3.2) a day, while SMS came from a mean of 12 patients (SD 4.4) per day. UPHS patients exposed within the hospital were asked to coordinate with the Hospital Infection Control Unit (HICU) for risk assessment. On the other

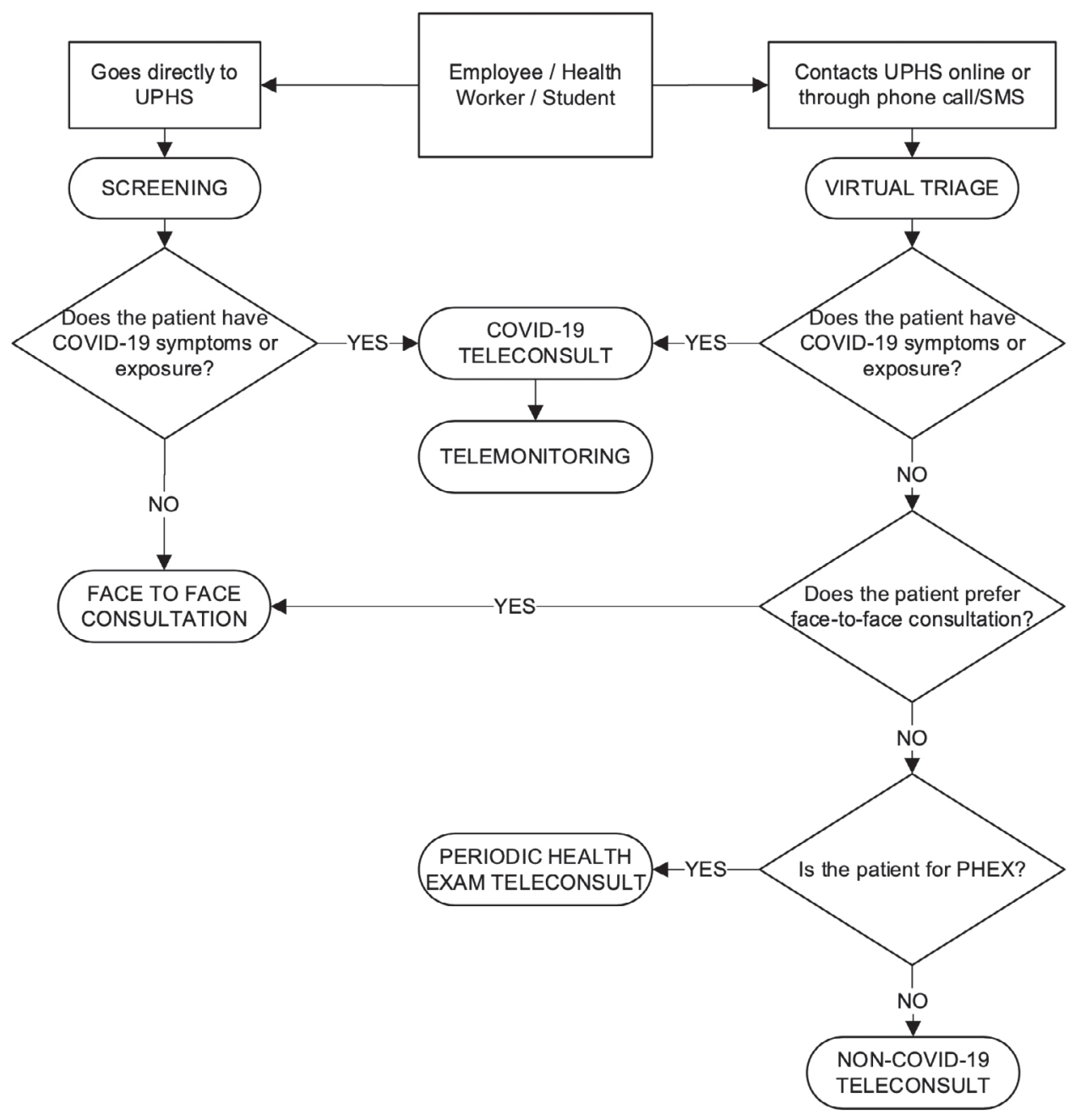

Figure 1. Patient Consultation and Monitoring Services in the UP Health Service using Telemedicine. 
Table 1. UPHS telemedicine census from October 18-31, 2020 during the COVID-19 pandemic

\begin{tabular}{lcr}
$\begin{array}{l}\text { Telemedicine Mode/Subclinic } \\
\text { Virtual Triage (number of messages/calls/online appointment } \\
\text { requests received) }\end{array}$ & $\begin{array}{l}\text { Total } \\
\text { rerage (SD) per day }\end{array}$ \\
\hline SMS* & 698 & $50(23.3)$ \\
Email $_{\text {OCRA }}^{\dagger}$ & 362 & $26(6.8)$ \\
Voice Calls & 261 & $26(11.6)$ \\
\hline Telemedicine Sub-clinic (number of patients seen) & $10(5.2)$ \\
\hline Telemonitoring & 129 & $155(40.8)$ \\
COVID-19 consults & $2,158^{\ddagger}$ & $21(6.4)$ \\
Non-COVID-19 consults & 292 & $9(3.4)$ \\
PHEX & 89 & $<1(0.4)$
\end{tabular}

* SMS, Short messaging service

${ }^{\dagger}$ OCRA, Online Consultation Request and Appointment

* Total number of patients seen in telemonitoring is the cumulative number of monitoring activities during the two-week period and is not equivalent to the number of unique individuals monitored.

${ }^{\S}$ PHEX, Periodic health examination

hand, employees' messages or calls with non-COVID-19 related complaints were scheduled for a teleconsult or faceto-face clinic visit depending on patient preference and chief complaint. SMS with non-COVID-19 concerns came from a mean of four patients (SD 3.5) per day.

Employees who came in person to the UPHS for laboratory requests through RADISH were also directed to non-COVID-19 teleconsult. The virtual triage nurse forwarded patient contact information to the telemedicine sub-clinics and instructed the patient to wait for a call, both for patients who were unable to email a CIF and for nonCOVID-19 teleconsult patients.
Appointment requests that were scheduled and triaged via OCRA were at a daily average of 26 (SD 11.6). COVID-19 teleconsults had a mean of two (SD 1.5) requests per day, non-COVID-19 consult had five (SD 3.3), and eight (SD 3.0) for other services, which include sick leave authentication, vaccination and prescriptionfilling. However, $83 \%$ of the appointment requests for other services were mistriaged requests for COVID-19 or nonCOVID-19 consultations.

\section{Non-COVID-19 Teleconsultations}

Teleconsultations for non-COVID-19-related medical concerns were conducted with 89 employees and students with an average of nine consults (SD 3.3) per day. Among the teleconsultations done, only $55 \%$ requested an appointment via OCRA. There were eight non-COVID-19-related emails received, containing documents from students for periodic health examination (PHEX) and pertinent photographs from patients for teleconsultation.

The consultation process started with employees and students being scheduled for non-COVID-19 teleconsult and endorsed by the triage nurse to the consultant-on-duty. Assessments and management were based on employees' medical and psychosocial concerns. Patients were advised to send photographs of affected areas and laboratory results, as applicable for their specific problems. All non-COVID-19 teleconsultations were encoded in RADISH. Electronic copies of the diagnostic requests and prescriptions were emailed to the patient, as necessary. Patient referral to a specialty clinic was done by the consultant or the triage nurse through OCRA.

PHEX was also conducted for two students via voice call and both were scheduled via OCRA. Required documents

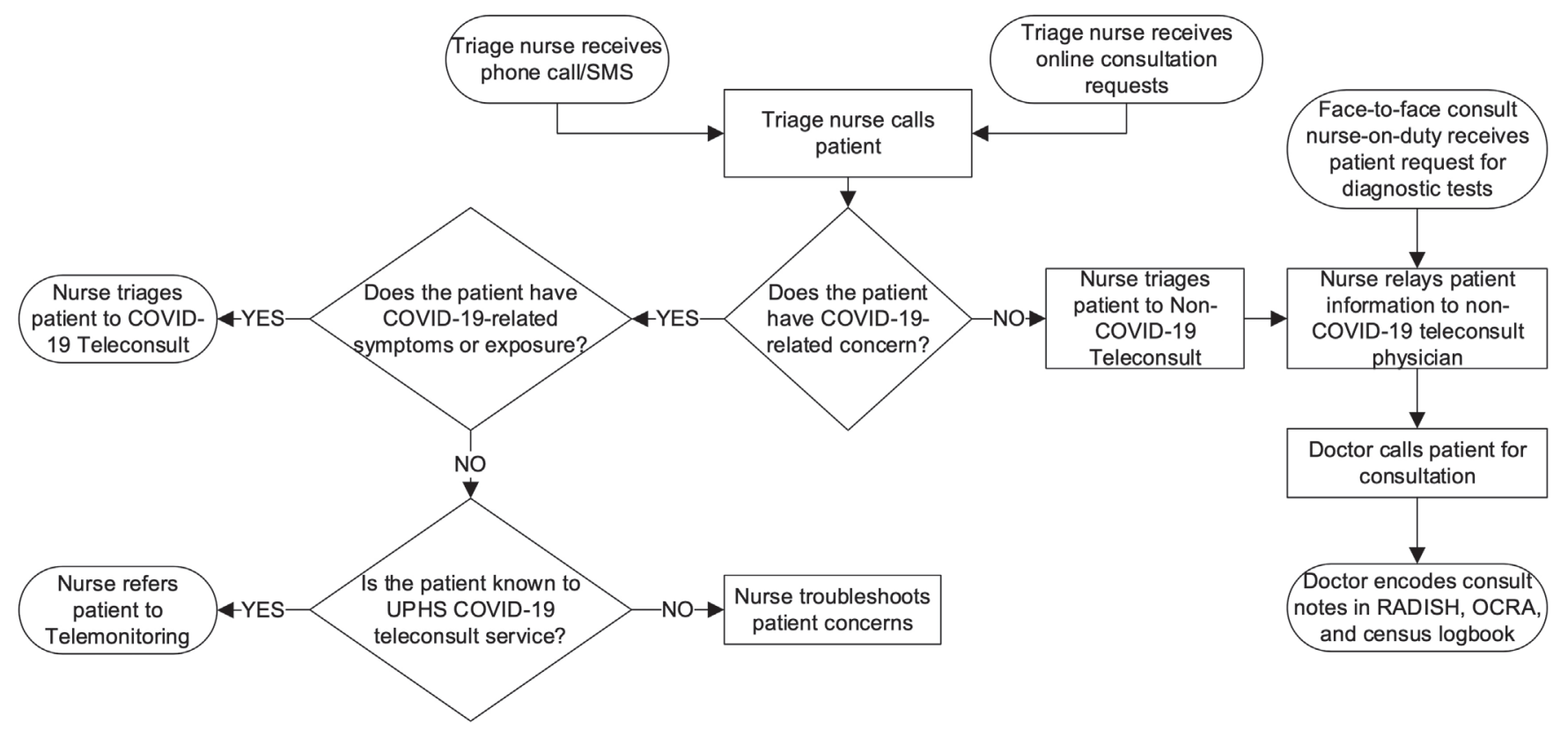

Figure 2. UPHS Telemedicine Process Flows for Virtual Triage and Non-COVID-19 Teleconsults. 
were assessed by the designated PHEX resident. Students with complete documents were allowed to proceed with the evaluation, while patients with comorbid conditions were referred to the appropriate specialist for co-management. The students and their respective colleges were informed of the medical evaluation results.

\section{COVID-19 Teleconsultations}

A total of 292 COVID-19-related consults were attended through emails and/or voice correspondence (Table 1). A resident physician monitored the UPHS email for COVID-19-related messages and CIF attachments during the eight-hour duty shift. Eighty-seven percent of COVID19-related consults with CIF were sent through email, while $13 \%$ provided CIF data through voice calls. The COVID-19 teleconsult subclinic responded to a total of 262 emails with an average of 19 (SD 7.5) per day.

Employees who were classified as COVID-19 suspects and those with high-risk exposure were scheduled for RTPCR testing. On the other hand, asymptomatic patients with exposure were referred to HICU for risk stratification before COVID-19 teleconsult. All patient encounters were documented in the electronic medical records (RADISH and ERP). Prescriptions, diagnostic requests or medical certi- ficates were sent to patients via email. The teleconsultation process for COVID-19 sub-clinic is illustrated in Figure 3.

\section{Telemonitoring services}

All employees and students who consulted for COVID-19 symptoms or exposure, including those who reported a positive RT-PCR test done at another facility, were enrolled for telemonitoring. An average of 155 patients (SD 40.8) were remotely monitored daily for progression or resolution of symptoms by SMS.

The service interventions were guided by patient symptoms. Medications were prescribed and additional laboratory tests and imaging were requested when necessary. Cases that required hospital admission were referred to the PGH Command Center. The mean number of patients with status updates per day was 71 (SD 10.4), which was $46 \%$ of the daily average number being actively monitored. Clinical management notes were encoded in the electronic medical records.

Telemonitoring was discontinued when employees were cleared as fit to work and has completed the quarantine period. A total of 220 patients finished active monitoring during the two-week period, averaging at 16 (SD 3.8) per day.

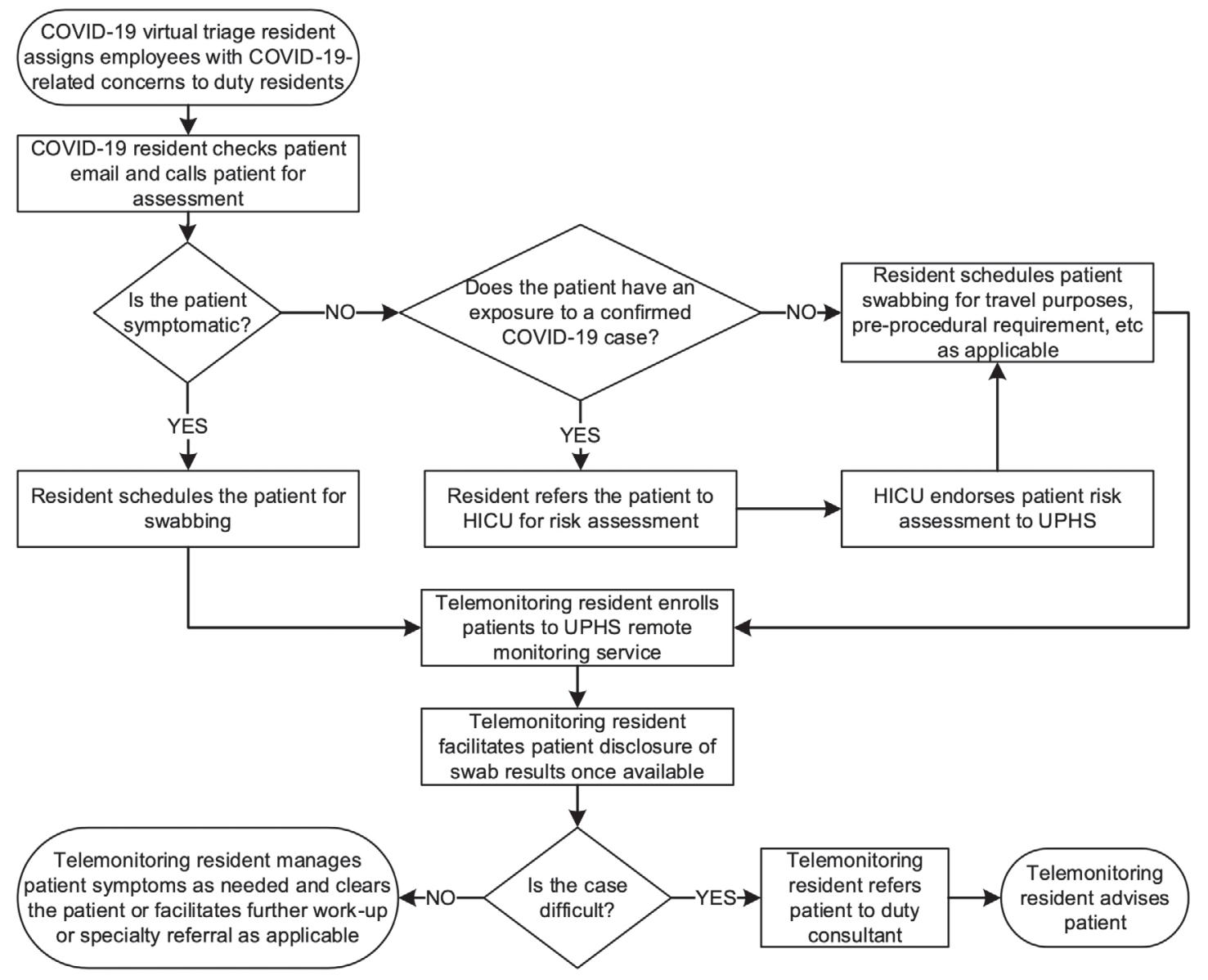

Figure 3. UPHS Telemedicine Process Flows for COVID-19 Teleconsults and Telemonitoring. 


\section{Good Telemedicine Practices}

Digital and online applications were used to expedite messaging and monitoring activities in the UPHS. SMS, phone calls, and emails were used as communication channels with patients and with other hospital units. Consultations via these applications were documented in an online database. Color coding was maintained as reference for the status of patients (e.g., red for COVID-19 confirmed, gray for those already cleared as fit to work). Updating and checking of the monitoring database were done regularly, and cases that needed validation were referred for consultation.

There was good coordination and constant communication among physicians between consultations. A consultant-on-duty facilitated referrals of difficult cases while the month-long rotation of staff-on-duty allowed familiarity with existing protocols. Monthly online endorsement was also conducted among residents for the shifting-in duty team. An endorsement document was also maintained for physicians on duty in the different sub-clinics.

Staff assigned in the triage area sent a standard reply to email inquiries (i.e., for COVID-19-related consultations and requests for RT-PCR swab), which outlined detailed instructions on filling-out the CIF as well as expected turnaround times. Staff were expected to be knowledgeable in current COVID-19 UPHS protocols. SMS messages were sent by the triage nurse to confirm appointments of patients scheduled via OCRA. There was constant communication among staff to update new policies, discuss issues encountered, and guide team members on duties and responsibilities. Multiple channels were used by the staff, including verbal/ electronic messaging (SMS, Do It Later app or Google Messages), phone calls and visual reminders (posters on steps/procedures) to facilitate clinic processes.

The RADISH system was utilized for maximum efficiency during consults. Laboratory results could be viewed in RADISH and e-signatures were utilized to streamline sending prescriptions. Reduction of rework was also facilitated by verifying diagnostics that could be done in PGH. The non-COVID-19 consultants also aided in providing fit-towork clearance of employees. The telemonitoring database was reviewed for clearance of patients and subsequent discontinuation from telemonitoring.

Physical distancing was also observed in the area with a maximum of two people per workstation.

\section{Areas for Improvement}

There was low utilization of the non-COVID-19 teleconsultation service since some patients preferred inperson consultation. Teleconsultations were done only through voice calls, since video calls were not feasible considering the clinic facilities. The lack of standard protocol in requesting for appointment, addressing unanswered calls, and documentation of consultation both in the logbook and electronic medical records caused disruption of workflow and service efficiency. Cellular provider policies interrupted service delivery, since outgoing phone calls were automatically disconnected after 12 minutes.

Inadequate patient knowledge of procedures and protocols, such as sending a complete CIF and the requirement of reporting exposure to HICU prior to consultation, caused delay in the COVID-19 tele-consultation process. The lack of coordination between HICU staff and the COVID-19 teleconsultation team decreased the efficiency in identifying and scheduling close contacts for swabbing. The limited number of computers also hindered better service delivery with one computer for consultants' teleconsultations, two computers for telemonitoring, three for COVID-19-related consultations, two for nurses and encoders. Four of the physicians assigned to COVID-19-related consultations and two clinic nurses brought their own laptops to augment the number of available devices. Incomplete data such as case number, contact details, and date of absence and quarantine period delayed the processing of patient enrolment to telemonitoring.

The use of OCRA in scheduling consults was not mandatory for all employees and students. Employees who used OCRA often made duplicate appointment requests. There were also patients who requested consultation through OCRA and were scheduled for a teleconsult but proceeded with face-to-face clinic visits leading to OCRA entries without disposition. New triaging processes or policies were not well-disseminated to staff members, while the quick turnover of staff also indicated the need for continuous orientation of personnel. Mistriages delayed the clinic process and provision of appropriate services.

Duty residents' tardiness was also identified as an area for improvement. Weekend duty residents were unfamiliar with some telemonitoring processes, such as importing contact information to the telemonitoring database and clinic phones, which led to carrying over of tasks to the next duty resident.

\section{DISCUSSION}

Triaging, consultations and monitoring were the main services being provided by the UPHS telemedicine service to hospital workers, university employees and students. The process of a clinic visit started with virtual triaging conducted by a staff nurse based on the health concern presented. Teleconsultation was composed of the COVID-19 subclinic that catered to patients with symptoms and exposure, and the non-COVID-19 sub-clinic that attended to patients with other medical concerns. Patients seen in the COVID-19 sub-clinic were enrolled in the telemonitoring service for remote monitoring of symptoms during home quarantine period.

Telemedicine is a crucial service during a pandemic to ensure that infection control measures are observed while addressing the medical needs of employees. It allowed access to health care services and information when prevention 
of infection spread by distancing was important. Nearly 4,000 telemedicine services were provided by UPHS during the study period of 14 days. In addition, UPHS was able to screen asymptomatic employees while assisting symptomatic quarantined individuals through remote monitoring.

Mobile messaging was a significant part of the monitoring of symptoms among confirmed/suspected COVID-19 employees during home-quarantine in order to detect progression of the disease or improvement in status. It enabled patients to access health services and directly communicate with physicians. Electronic mails, on the other hand, were the main entry portal of employees suspected of COVID-19-related symptoms or exposure. This reduced the number of individuals visiting the clinics, consequently avoiding facility overcrowding and potential spread of SARS-COV-2. Thus, telemedicine provided a safer environment for patients and healthcare workers by reducing possible contact with infected individuals while providing medical services when physical consultation is limited.

The World Health Organization (WHO) defined telemedicine as the use of information and communication technology to bridge geographical barriers, for the benefit of providing clinical support and improving health outcomes. It was proven to be effective in reaching patient populations with various specific needs. ${ }^{4}$ Web-based interventions and focused text messaging (SMS) were effective in improving weight, glycemic control, and cholesterol levels among individuals with type 2 diabetes., ${ }^{5,6}$ Likewise, the use of interactive education among HIV-infected patients showed better treatment compliance with use of telemedicine. ${ }^{7}$ During pandemics, telemedicine can also be utilized to screen, follow-up, monitor, and allow patients to access health facilities. ${ }^{2}$

Health care workers, who are at highest risk of infection due to occupational-related factors, can benefit from telemedicine as patients. A US hospital used a telemedicine platform in its occupational medicine clinic, receiving consultations for acute concerns, follow-up visits and periodic health examinations of facility workers. ${ }^{8}$ The use of telemedicine during the COVID-19 pandemic has also been reported in a few studies showing the use of various applications such as WeChat, SMS, and videoconferencing. ${ }^{6,9,10}$ However, there was a scarcity of data evaluating quality of care provided to urgent care patients through telemedicine. There are also concerns that telemedicine provides a variable quality of care including the absence of a physician-patient relationship, limited access to medical records, limitations on virtual physical examination and barriers to diagnostics which can lead to misdiagnosis or poor quality of care. ${ }^{11,12}$

The proper implementation of telemedicine, however, requires a well-functioning Information and Communications Technology (ICT) infrastructure. The cost of service maintenance and monitoring, the need to set up protocols for obtaining patient personal information, guidelines to ensure security and confidentiality, regulations and systems to monitor and limit data access, use and disclosure are additional challenges that need to be addressed for widespread adoption of telemedicine for health care. ${ }^{13,14}$

There are also physician- and patient-related barriers to the general acceptance of telemedicine. Limited access and literacy in the use of electronic devices are the most commonly cited patient-related barriers. The lack of familiarity in using the internet and awareness of the online platform were the main reasons reported by Zhang in 2014 for the low utilization (17\%) of an outpatient web-based appointment system (WAS) in a tertiary public hospital. ${ }^{15}$ This is consistent with the study finding that only $55 \%$ of teleconsultations conducted in UPHS had an online consultation appointment request. Physicianrelated barriers, on the other hand, include discomfort with clinical decision-making without a complete physical examination, technical difficulties in using electronic medical records for documentation, and lack of rapport-building through telemedicine. ${ }^{16,17}$

The UPHS was able to address the various health needs of employees during the COVID-19 pandemic, including the usual primary care consults and periodic examination of students and employees through the telemedicine services. The clinic, however, has been challenged to respond to the rapidly evolving requirements of multiple stakeholders during this COVID-19 pandemic. Telemedicine services were implemented and modified to be able to respond to the specific needs and policies of the hospital.

The study results are limited to the clinic services as part of the UPHS response to the pandemic. The processes may not be applicable in a scenario of post-COVID-19 UPHS wherein consultations may be more on chronic or noncommunicable diseases. Likewise, the telemedicine services were based on specific threats of the infectious nature of SARS-COV-2 and may evolve as the global knowledge and understanding of COVID-19 increases. The UPHS telemedicine processes are also limited by the hospital's present infrastructure and technological capacity. The design of the clinic's telemedicine process flow corresponds with the available hardware and software and may be different with systems used in other settings with more advanced ICT.

Study recommendations include transitioning telemedicine services into a permanent component of the clinic's services to include the regular monitoring and follow-up of employees with chronic conditions. Telemedicine can be a regular part of the department's residency training, clinical rotations, evaluations and curriculum. The upgrading of the clinic's technological capacity is also important in order to utilize video conferencing for telemedicine consultations and aid the assessment and management of UPHS patients. A dedicated telemedicine lane for the annual physical examination of employees may also be pilot-tested as the employees can be initially assessed through voice calls prior to in-person clinic consultations. The infrastructure 
upgrade of the telemedicine services can also be a venue for evaluation of the quality of care provided by UPHS through audio and video recordings.

\section{CONCLUSION}

Telemedicine services at the UPHS included teletriaging, teleconsultations, and telemonitoring with use of phone calls, SMS, emails, and OCRA. Timely coordination, on-site duty consultants, and use of technology had been identified as good practices. Lack of protocols and inconsistent OCRA use are areas for improvement.

\section{Statement of Authorship}

All authors participated in the data collection and analysis and approved the final version submitted.

\section{Author Disclosure}

All authors declared no conflicts of interest.

\section{Funding Source}

None.

\section{REFERENCES}

1. Centers for Disease Control and Prevention. Using Telehealth to Expand Access to Essential Health Services during the COVID-19 Pandemic [Internet]. 2020 June 10 [cited 2021 January]. Available from: https://www.cdc.gov/coronavirus/2019-ncov/hcp/telehealth. html

2. Ohannessian R. Telemedicine: potential applications in epidemic situations. Eur. Res. Telemed. 201; 4:95-8. doi: 10.1016/j.eurtel.2015. 08.002 .

3. Serrat O.Identifying and Sharing Good Practices. Knowledge Solutions [Internet]. 2008 [cited 2021 January]. Available from: https://www. adb.org/sites/default/files/publication/27598/identifying-sharinggood-practices.pdf

4. Kruse CS, Karem P, Shifflett K, Vegi L, Ravi K \& Brooks M. Evaluating barriers to adopting telemedicine worldwide: a systematic review. J Telemed Telecare, 2018; 24(1):4-12. doi: 10.1177/1357633X16674087

5. Bond GE, Burr R, Wolf FM, Price M, McCurry SM, Teri L. The effects of a web-based intervention on the physical outcomes associated with diabetes among adults age 60 and older: a randomized trial. Diabetes Technol Ther. 2007 Feb; 9(1):52-9. doi: 10.1089/dia. 2006.0057.
6. Haider R, Sudini L, Chow CK, Cheung NW. Mobile phone text messaging in improving glycaemic control for patients with type 2 diabetes mellitus: A systematic review and meta-analysis. Diabetes Res Clin Pract. 2019 Apr; 150:27-37. doi: 10.1016/j.diabres.2019. 02.022 .

7. Gras G. Use of telemedicine in the management of infectious diseases. Médecine et Maladies Infectieuses. 2018; 48(4):231-7.

8. Varkey P, Hagen PT, Wimsett W, Buchta W. Telemedicine applications in occupational medicine. Minn Med. 2006 Nov; 89(11):46-8.

9. Xu H, Huang S, Qiu C, Liu S, Deng J, Jiao B, et al. Monitoring and management of home-quarantined patients with COVID-19 using a WeChat-based telemedicine system: retrospective cohort study. J Med Internet Res. 2020 Jul 2; 22(7):e19514. doi: 10.2196/19514.

10. Reuter E. Johns Hopkins rolls out COVID-19 monitoring tool for healthcare workers [Internet]. March 24, 2020 [cited 2021 January]. Available at https://medcitynews.com/2020/03/johns-hopkins-rollsout-covid-19-monitoring-tool-for-healthcare-workers/

11. Halpren-Ruder D, Chang AM, Hollander JE, Shah A. Quality assurance in telehealth: adherence to evidence-based indicators. Telemed J E Health. 2019 Jul; 25(7):599-603. doi: 10.1089/tmj. 2018.0149.

12. Uscher-Pines L, Mulcahy A, Cowling D, Hunter G, Burns R, Mehrotra A. Access and quality of care in direct-to-consumer telemedicine. Telemed J E Health. 2016 Apr;22(4):282-7. doi: 10.1089/tmj.2015.0079.

13. Combi C, Pozzani G, Pozzi G. Telemedicine for developing countries. a survey and some design issues. Appl Clin Inform. 2016 Nov 2; 7(4):1025-50. doi: 10.4338/ACI-2016-06-R-0089.

14. Wright J, and Caudill R. Remote treatment delivery in response to the COVID-19 pandemic. Psychother Psychosom 2020; 89:130-2. doi: $10.1159 / 000507376$

15. Zhang M, Zhang C, Sun Q, Cai Q, Yang H, Zhang Y. Questionnaire survey about use of an online appointment booking system in one large tertiary public hospital outpatient service center in China. BMC Med Inform Decis Mak. 2014 Jun 9; 14:49. doi: 10.1186/14726947-14-49.

16. Barney A, Buckelew S, Mesheriakova V, Raymond-Flesch M. The COVID-19 pandemic and rapid implementation of adolescent and young adult telemedicine: challenges and opportunities for innovation. J Adolesc Health. 2020 Aug;67(2):164-171. doi: 10.1016/ j.jadohealth.2020.05.006.

17. Tenforde AS, Iaccarino MA, Borgstrom H, Hefner JE, Silver J, Ahmed M, Babu AN, Blauwet CA, Elson L, Eng C, Kotler D, Homer S, Makovitch S, McInnis KC, Vora A, Borg-Stein J. Telemedicine during covid-19 for outpatient sports and musculoskeletal medicine physicians. PM R. 2020 Sep;12(9):926-932. doi: 10.1002/pmrj.12422. 